\title{
Bumpy Patches: Analgesic Effects of Particle Pressure in Sports Injury Treatment
}

\author{
Norio SAIto, ${ }^{* * * *, ~ \# ~ G e n ~ K a n e K o, ~}{ }^{* *}$ Kazuyuki Mito ${ }^{* * *}$
}

\begin{abstract}
Physical analgesia has been used for the treatment of sports injuries. We previously developed a special patch (bumpy patch, BP) that adds pressure to the pain area, demonstrating its analgesic effects in various sports injuries. In this study, we further verified acute and chronic analgesic effects of the BP in 28 subjects (14-44 years of age, 15 males and 13 females) with various pain sites and histories of pain (knee, shoulder, low back, elbow and other joints; 6 months to 3 days). First, the trigger area with muscle induration or tenderness was identified in each subject by palpation, and each subject evaluated the degree of pain using the numerical rating scale (NRS). Then the BPs were applied to completely cover the pain area. The BP treatment was repeated at 2 to 3-day interval until all subjects reported no pain (NRS $=0$ ), and the differences between pre- and post-treatment NRS values were compared for each treatment as the measure of the analgesic effect. Significant analgesic effects were observed in the first and second BP treatments $(P=0.000774$ and 0.00149 , respectively). The third BP treatment also reduced the pain intensity, but the difference was not statistically significant $(P=0.3734)$. Some subjects reported mobilization of the pain area after the BP treatment, which was discussed in relation to the potential analgesic mechanism of the BP. These results indicate that the BP has acute and chronic analgesic effects and can be used for the treatment of sports injuries.
\end{abstract}

Keywords: athlete, analgesia, particle pressure, healing effect.

Adv Biomed Eng. 10: pp. 123-128, 2021.

\section{Introduction}

Traditional sports injury treatments includes surgery, nerve block treatment (opioid injection), drug injection to trigger points, and acupuncture and moxibustion treatment [1-4]. Although these approaches have shown efficacy in the treatment of sports injuries, they sometimes require a long treatment period depending on the severity of the injury. Long-term withdrawal from the game not only impairs athletes' intuition, but also may threaten their career. Therefore, there is a continuous need to de-

Received on May 31, 2021; revised on August 30, 2021; accepted on September 9, 2021.

* Acupuncture and Moxibustion Traditional Medicine Clinic, Shizuoka, Japan.

${ }^{* *}$ School of Arts \& Sciences, University of Houston-Victoria, Victoria, USA.

*** The Graduate School of Informatics and Engineering, The University of Electro-Communications, Tokyo, Japan.

\# Takasawa-cho 3-15 Numazu, Shizuoka 410-0057, Japan. E-mail: ryu9ten7@yahoo.co.jp

\section{(c) BY}

Copyright: (02021 The Author(s). This is an open access article distributed under the terms of the Creative Commons BY 4.0 International (Attribution) License (https:// creativecommons.org/licenses/by/4.0/legalcode), which permits the unrestricted distribution, reproduction and use of the article provided the original source and authors are credited. velop a method that produces effective pain relief in a short treatment time.

Physical analgesia has been used in various countries as one of the treatments that exerts an analgesic effect $[5,6]$. In addition to traditional physical analgesic therapies such as electrotherapy and thermotherapy, pyramidal thorn patches have been used for pain relief, although the application has been largely limited to Japan. Figure 1 shows a type of pyramidal thorn patch, designated the bumpy patch (BP; product name Athlete-Aid; $7 \mathrm{~mm}$ square at the bottom, $3 \mathrm{~mm}$ in height; Super Medical Japan, Co. Ltd., Numazu, Japan) hereafter, invented by Saito et al. in 2018 [7, 8]. In contrast to the traditional pyramidal thorn patches that are directly placed on the pain area, the BPs are placed to target a wide area surrounding the point of pain. The thorn patches have ad-

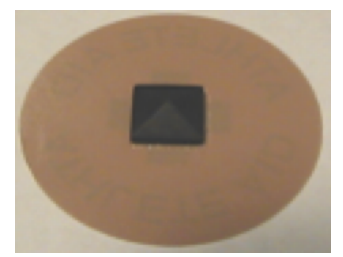

Fig. 1 A bumpy patch (BP) with a pyramid-shape particle (7 $\mathrm{mm}$ square at the bottom, $3 \mathrm{~mm}$ in height). The adhesive material has a diameter of $3 \mathrm{~cm}$. 
vantages over other physical analgesia in that they can be applied to patients in a short treatment time without special equipment, and that the analgesic effect may last longer since patients do not need to remove the patches outside the hospital. However, physical stimulation treatments such as BPs have been categorized as an "alternative medicine," which is not part of standard medical care, although there is an increasing interest in physical therapies in Western countries $[9,10]$. This is partly because the analgesic mechanism of physical analgesia remains unknown, although it is hypothesized that gentle physical stimulation elicits nervous signal that alleviates pain sensation [11]. The accumulation of more clinical evidence from the mechanistic insight is warranted to develop thorn patches into an established medical practice.

Among the patch-based treatments, the BP treatment has been relatively well validated for the analgesic effects. Saito et al. [7] reported the analgesic effect of one-time BP treatment measured by the visual analog scale (VAS) by studying approximately 300 patients with various sports injuries. Near-infrared spectroscopy (NIRS) also confirmed the effect of BP. This device noninvasively visualizes dynamic changes in oxyhemoglobin and deoxyhemoglobin concentrations in the brain, illustrating the pain- and itch-specific patterns of blood flow responses [12]. The BP treatment caused a reduction of pain-specific NIRS pattern, providing an objective measure of the patients' pain perception. The BP treatment therefore has the potential to be a target of future mechanistic research, which is largely unexplored in this field. In this study, we aimed to accumulate more evidence for the analgesic effect of BP. In contrast to the previous study [8], the effect of multiple treatments was tested by following patients until they reported no pain. While the BP treatment may be effective for various types of pain, patients with sports injuries were recruited because of the high prevalence of sports injury among young people [8] and the special needs of athletes for effective treatments.

\section{Materials and Methods}

\subsection{Ethical statement}

This research was carried out at the Tokai Orthopedic Clinic based on the diagnosis by physicians at the Department of Orthopedic Surgery, Seisen Clinic between November 4, 2020 and December 15, 2020. All research procedures were conducted by Norio Saito, a specialist in the treatment of sprain and contusion certified by the Ministry of Health, Labor and Welfare, Japan (registration number .30309), based on the Judo Rehabilitation Law No. 49. The research protocol was approved by the University of Electro-Communications Ethics Committee (No. 20029) and registered in the Japanese database of clinical trials, UMIN Clinical Trials Registry (Ref 2020 UMIN ID: 000042796). The investigation was conducted in accordance with the principles set out in the Declaration of Helsinki. Subjects were recruited from patients of the Tokai Orthopedic Clinic. Signed consent forms were obtained from all subjects after fully explaining the purpose of the research and procedure of the treatment. There was no compensation or billing for participation.

\subsection{Subjects}

This study included a total of 28 subjects (15 males and 13 females) with different durations of sports injury: 3 subjects with 6 to 3 months, 10 with 3 months to 3 weeks, and 15 with 3 weeks to 3 days. The age range was 14-44 years with an average of 23.5 years. The subjects were requested to follow the guidelines: 1) take a break for 15 to $30 \mathrm{~min}$ after treatment; 2) do not watch TV, play games, or read books until they leave the study because these activities often cause eye strain and posture-related symptoms such as sore neck [13], which may negatively affect the treatment outcome; 3 ) do not exercise vigorously (mild movement of muscles and joints is permitted as pain management); and 4) perform self-pain check three times after a BP treatment, in which they test whether any posture or activity normally occurring in the sport causes the pain. Subjects received the BP treatment at an interval of a few days until they felt no pain (NRS = $0)$ at the self-pain check.

\subsection{Evaluation of the analgesic effect}

In order to evaluate the analgesic effect of BPs, the subjects reported the degree of pain before and after the treatment using the numerical rating scale (NRS). The pain assessment was performed by a non-operator to minimize the patients' care bias towards the practitioner. A single non-operator conducted all assessments to remove any observer bias.

The first pre-treatment pain intensity was determined as follows. The subject maintained the posture that caused the most severe pain, and the induced pain was defined as "NRS $=100$." During the pre-treatment assessment, the operator identified the pain area using a finger at the posture that induced the pain. Specifically, the site of pain was identified by palpation as reported previously [14] using the traditional signs of inflammation such as tenderness and induration $[15,16]$. When the subject reported "tenderness," which is a type of pain induced by touching, the pain area was identified and marked by pushing all the surrounding areas (Fig. 2A, B). A pain detection rod (Fig. 3; Toutsu Ryouiki Tansa Bou, Otsuka Tekkoujo, Co. Ltd., Numazu, Japan) was used to determine the boundary of the pain area when 

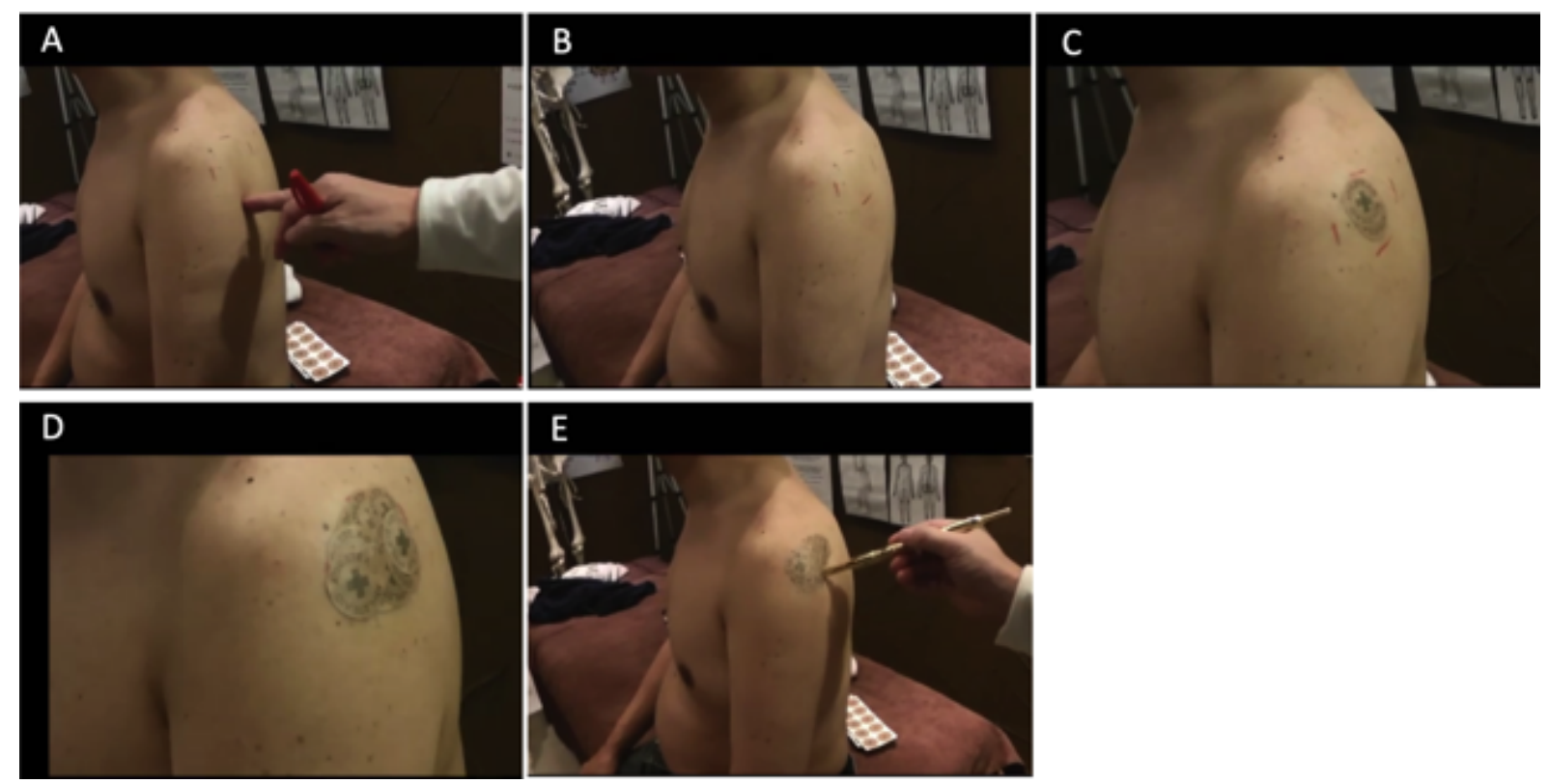

Fig. 2 Identification of the pain area and BP treatment. The operator identified (A) and marked (B) the pain area. BPs were placed on the shoulder to completely cover the pain area $(\mathrm{C})$. When the pain area was larger than a BP, multiple BPs were used for one pain area (D). When the boundary of the pain area was not clear, a pain detection rod was used to identify the boundary $(\mathrm{E})$.

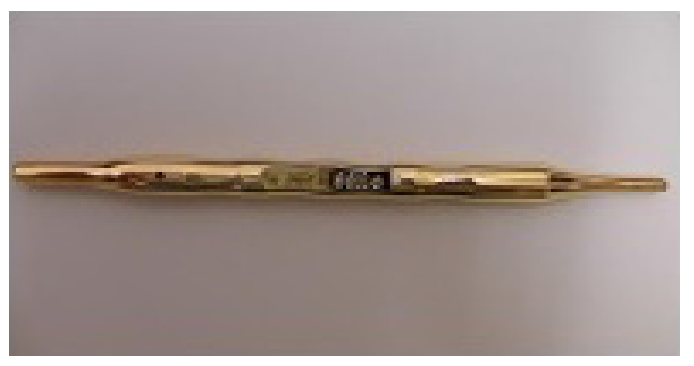

Fig. 3 Pain detection rod used to determine the pain trigger area. The rod has a length of $20 \mathrm{~cm}$, weight of $150 \mathrm{~g}$, and two ends with diameters of $7 \mathrm{~mm}$ (left) and $5 \mathrm{~mm}$ (right). The 7-mm diameter end is used for most injuries. The 5-mm diameter end was used for small pain areas.

palpation could not clearly indicate the boundary. The operator's finger was used instead of the pain detection rod for identifying the pain area when the pain was categorized as muscle induration or tender pain with muscle induration, or when the subject was sensitive to pain.

Subsequently, the BPs (Fig. 1) were applied onto the skin, such as that the convex part of the BPs completely covered the entire pain area (first treatment; Fig. 2C and 2D). The first post-treatment pain intensity was measured immediately after the first treatment ( 3 to $5 \mathrm{~min}$ after placing the BPs). The difference in the first pre- and post-treatment pain intensity values, represented by the NRS, was regarded as the analgesic effect of the BP. The
BPs were removed when patients felt itching (typically up to 12 hours, maximum 24 hours).

Within three days from the first treatment described above, subsequent treatments were conducted as follows. The treatments were continued at 2- to 3-day interval until the subject reported no pain. This interval was set in order to treat pain recurrence, which takes place after 2-3 days of the treatment, based on our experience. The procedure for subsequent treatments was the same as that of the first treatment, and the pre- and post-treatment pain intensities were recorded for each treatment. Two subjects showed skin rash, but no other side effects were observed. Wilcoxon signed rank test was used to detect significant differences between pre- and post-treatment NRS values.

\section{Results}

The overall treatment schedule for all subjects is summarized in Fig. 4. A total of 30 candidate subjects were enrolled in the study. After excluding 2 candidates (lack of data), 28 subjects participated in the treatment study. The initial average NRS of the 28 subjects was 58.3. There were no significant differences in NRS among subject groups with different pain durations and with different pain sites.

The 28 subjects had different pain durations: within 3 days $(n=5), 1$ week $(n=4), 2$ weeks $(n=2), 3$ weeks $(n=2), 1$ month $(n=4), 3$ months $(n=8)$, and 6 months $(n=3)$. In addition, 17 subjects had acute pain, whereas 


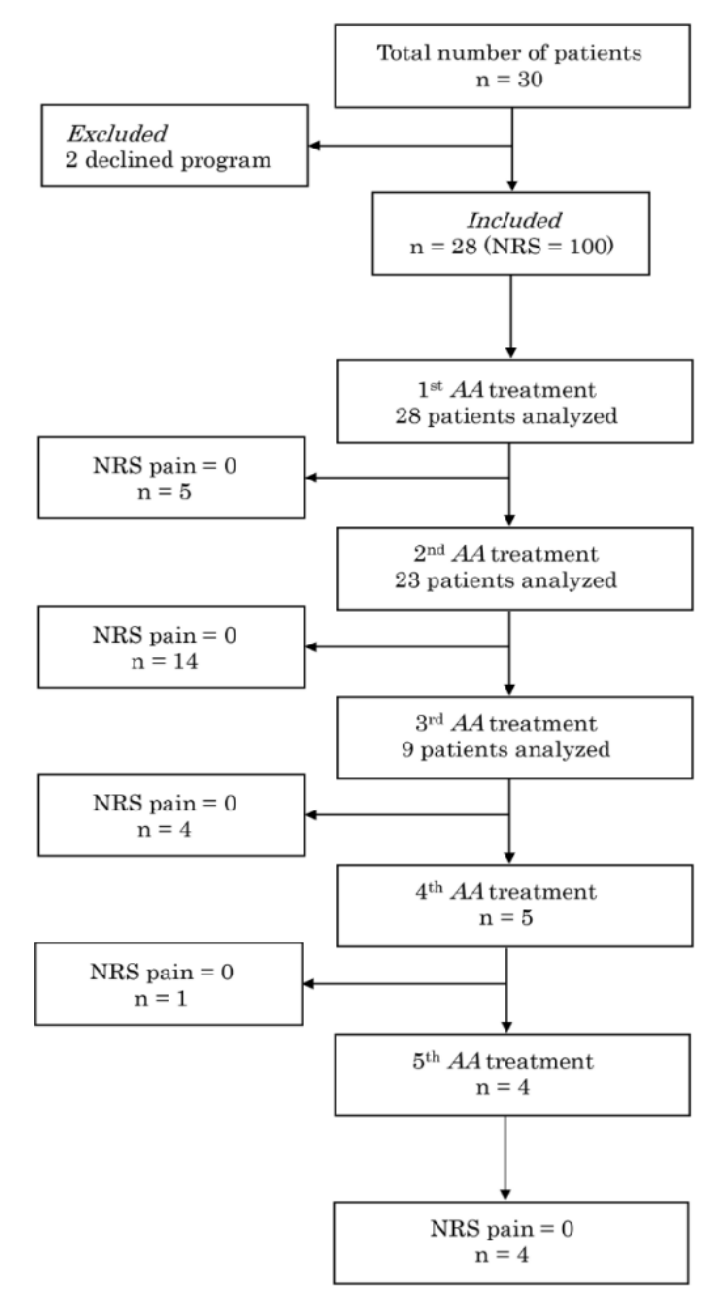

Fig. 4 Data analysis and exclusion flowchart. Subjects continued to receive BP treatment at 2 to 3-day interval until they reported no pain $(\mathrm{NRS}=0)$. The final assessment of pain was conducted at the posture that causes the most severe pain. In the case of knee pain, for example, deep bending, stretching, fast running, and jumping were tested.

11 had chronic pain. Five treatments were required until all subjects reported no pain (NRS $=0$ ) and left the study (Fig. 4). Five subjects reported no pain after the first treatment, 14 after the second treatment, 4 after the third treatment, 1 after the fourth treatment, and 4 after the fifth treatment. The subjects with lower back pain and knee pain showed slower pain relief compared to the other subjects with movement of pain around the trigger area. There was no clear relationship between the duration of pain (6 months to 3 days) and the number of treatments required for pain relief. One-month after the study period, participants were asked over the phone if they had recurrence of pain. Seventeen of $28(60.7 \%)$ subjects reported no recurrence, while four reported recurrences and seven reported pain at different areas (movement of pain).

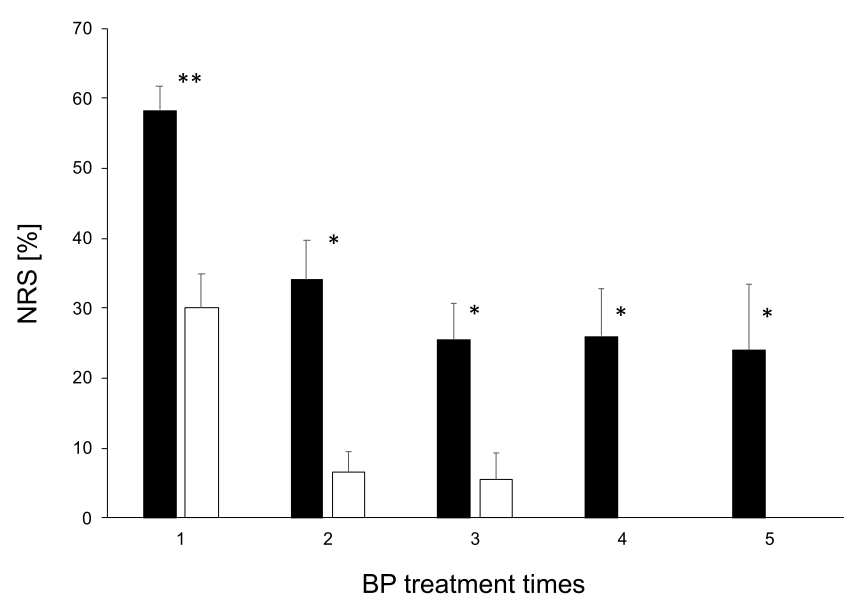

Fig. 5 Effect of successive BP treatments on the numeric rating score (NRS). Error bars of the first, second, third, fourth, and fifth treatments represent standard errors in 28, 23, 9, 5, and 5 subjects, respectively. ${ }^{* *} P<0.0001 ;{ }^{*} P<0.01$. No significant difference was detected between pre- and post-treatment NRS in the third treatment $(p=0.3734)$.

The BP treatment resulted in significant reduction of the pain intensity in the first (mean difference in NRS 28.32, $P=0.000774$ ) and second (mean difference in NRS 27.63, $P=0.00149$ ) treatments (Fig. 5). All subjects reported a positive analgesic effect of BP with the first treatment; first pre-treatment NRS values were higher than first post-treatment NRS values in all subjects, although subjects with a severe ankle sprain reported only minor recovery after the BP treatment. In contrast, the second pre-treatment NRS values were higher than the first post-treatment NRS values, and the third pre-treatment NRS values were higher than the second post-treatment NRS values. These results suggest a minor effect of natural recovery on the NRS values, at least in some subjects. The third, fourth, and fifth BP treatments also significantly reduced the pain intensity $(P=$ $0.00614,0.00499$, and 0.00542 , respectively), although the numbers of subjects who received these treatments were less than the first and seconds treatments.

\section{Discussion}

In this study, the first and second BP treatments significantly reduced NRS values in subjects with various sports injuries. The majority of subjects had pain relief (i.e., subjects reported NRS $=0$ ) within two or three days. These results are consistent with our previous study of 300 people with sprains [8], and likely indicate the analgesic effect of the BP.

When applying the BP, the pain trigger area was carefully identified, and BPs were applied to the entire trigger area. Most subject needed 20 or fewer BPs, but 
the number increased depending on the size of the trigger area $\left(1.2-2.2 \mathrm{~cm}^{2} / \mathrm{BP}\right.$ in order to make the distance between particles less than $3 \mathrm{~mm}$ ). For example, in a case of shoulder injury, a total of 64 BPs were applied to the pain site (front and back of the shoulder, deltoid and trapezius muscles, and back of the neck). Another example was a 15-year-old male with cruciate ligament rupture. A total of 520 BPs were applied on the back of the knee, medial, lateral, lower leg (soleus), quadriceps, affected lumbar and thoracic vertebrae. This is a marked difference between the BP treatment and conventional acupuncture. In conventional acupuncture, the stimulation focuses on the point of the pain, not the area of the pain, to improve the local blood circulation.

Several recent studies have demonstrated that gentle mechanical skin stimulation by microcones inhibits sympathetic pain responses via the $\mathrm{A} \delta$ and $\mathrm{C}$ fibers in rats [17, 18], which transmit sharp and dull pain, respectively. Microcones can also be applied on human skin using a disk, as in the case of BP, to attenuate heat-induced autonomic responses [19]. In contrast to the BP, however, the application of microcone discs does not significantly affect subjective pain measured by VAS. This difference may be attributable to the different strengths of mechanical stimulation between microcones and BP. The height of microcones is $0.3 \mathrm{~mm}[18,19]$, whereas the BP has a pyramid-shape particle $3 \mathrm{~mm}$ in height, which provides stronger mechanical stimulation. While both treatments are worthy to be further investigated for the mechanism and possibility of applications, the current body of data suggests that the BP has an advantage as a treatment method over microcones because of the analgesic effect on subjective pain.

There are various possible mechanisms by which the BP exerts an analgesic effect. In the case of acupuncture and moxibustion, various sensory stimulations caused by pressure, temperature, vibration, and acupressure are transmitted to the nervous system via sensory receptors. These sensory signals are transmitted by thick nerve fibers at a higher speed than pain signals transmitted by $\mathrm{A} \delta$ (sharp and fast pain) and $\mathrm{C}$ (dull pain) fibers, resulting in the suppression of pain signals [20]. This gate control theory well explains the analgesic effect of the BP as well as those of the microcones $[17,18]$ and Kinesio taping [21]. Furthermore, this theory may also explain the "mobilization of the pain area" after the BP treatment reported by some subjects. If the signals from the center of the pain area were suppressed by the BP treatment, subjects would feel pain signals from surrounding area more strongly, resulting in the feeling that the pain was "moved."

On the other hand, subjects in this study reported the analgesic effects even after the BPs were removed from the pain area. Since the gate control is in principle effective only when the sensory stimuli are present, other mechanisms may also be involved. The increased blood flow may serve as an underlying mechanism. It is known that the axon reflex relieves pain by increasing blood flow in response to the pain substances from the pain site [22]. In addition, increase in blood flow is the most common effects of physical massage therapy [23, 24]. Because it is a unique feature of the BP treatment to place many patches over the entire pain area, the blood flow of the area may have been increased during the treatment. This possibility can be tested by measuring the local skeletal muscle blood flow by NIRS. Alternatively, BPs placed on wide area may cause neurogenic inflammation, which in turn affects the skin mobility and exerts the analgesic effect. These potential mechanisms should be carefully considered since the prolonged analgesic effect could be an advantage of the BP over other sensory stimulation methods.

\section{Conclusion}

In this study, we demonstrated the analgesic effect of the BP in 28 subjects with various sports injuries. The acute analgesic effect of BP would help athletes shorten the treatment period, preventing muscle atrophy caused by long-term withdrawal from training. Future studies will investigate the mechanism of BP treatment by recruiting more subjects.

\section{Conflict of interest}

The authors declare no conflict of interest.

\section{Ethics declaration}

This research was carried out at the Tokai Orthopedic Clinic based on the diagnosis by physicians at the Department of Orthopedic Surgery, Seisen Clinic between November 4, 2020 and December 15, 2020. All research procedures were conducted by Norio Saito, a specialist in the treatment of sprain and contusion certified by the Ministry of Health, Labor and Welfare, Japan (registration number .30309), based on the Judo Rehabilitation Law No. 49. The research protocol was approved by the University of Electro-Communications Ethics Committee (No. 20029) and registered in the Japanese database of clinical trials, UMIN Clinical Trials Registry (Ref 2020 UMIN ID: 000042796). The investigation was conducted in accordance with the principles set out in the Declaration of Helsinki. Signed consent forms were obtained from all subjects after fully explaining the purpose of the research and procedure of the treatment.

\section{References}

1. Itoh K, Inoue T, Haneda Y, Ochi H, Kitakoji H: The effect of 
trigger point acupuncture treatment on sport-induced chronic low back pain. Questionnaire and acupuncture treatment for university students. (In Japanese with English Abstract). J Jpn Soc Acupunct Moxibust. 55, 142-149, 2005.

2. Figueroa D, Figueroa F, Calvo R: Patellar tendinopathy: diagnosis and treatment. J Am Acad Orthop Surg. 24(12), e184-e192, 2016.

3. Orlandi D, Corazza A, Arcidiacono A, Messina C, Serafini G, Sconfienza LM, Silvestri E: Ultrasound-guided procedures to treat sport-related muscle injuries. Br J Radiol. 89(1057), 20150484, 2016.

4. Malone M: The utility of acupuncture in sports medicine: a review of the recent literature. J Sports Med Ther. 2, 020-027, 2017.

5. Koleva I, Yoshinov B, Yoshinov R: Perspectives in pain management: physical analgesia. Med J Clin Trials Case Stud. 2, 000135 , 2018.

6. Liu D, Ma J, Zhang Z, Yu A, Chen X, Feng C, Lei W: Management of postoperative pain in medical institutions in Shandong Province in China. Medicine. 95(6), e2690, 2016.

7. Saito N, Shima R, Yamada Y, Nagaoka M, Ito E, Yoshioka T: A proposed molecular mechanism for physical analgesia in chronic pain. Neural Plast. 2018, 1260285, 2018.

8. Saito N, Shima R, Yen CT, Yang RC, Ito E, Yoshioka T: Adhesive pyramidal thorn patches provide pain relief to athletes. Kaohsiung J Med Sci. 35(4), 230-237, 2019.

9. Galantino ML, Boothroyd C, Lucci S: In Complementary and alternative medicine interventions for the orthopedic patient: a review of the literature, Seminars in Integrative Medicine, 2003; Elsevier, pp. 65-79, 2003.

10. Kearney G, Cioppa-Mosca J, Peterson MG, MacKenzie CR: Physical therapy and complementary and alternative medicine: an educational tool for enhancing integration. HSS J. 3(2), 198201, 2007.

11. Ito E, Shima R, Yoshioka T: A novel role of oxytocin: Oxytocininduced well-being in humans. Biophys Physicobiol. 16, 132139, 2019.

12. Lee C-H, Sugiyama T, Kataoka A, Kudo A, Fujino F, Chen Y-W, Mitsuyama Y, Nomura S, Yoshioka T: Analysis for distinctive activation patterns of pain and itchy in the human brain cortex measured using near infrared spectroscopy (NIRS). PLoS ONE. 8(10), e75360, 2013.

13. Sitzman K: Tips for managing computer vision syndrome. AAOHN J. 53(12), 556-556, 2005.

14. Saito N, Sakamoto K, Ota M, Kobayashi K, Mano T, Owa K: Analgesic effect of the metal grain sheet placed on the pain area (In Japanese with English abstract). J Jpn Soc Stud Chronic Pain. 27, 121-129, 2008.

15. Ling FW: Randomized controlled trial of depot leuprolide in patients with chronic pelvic pain and clinically suspected endometriosis. Obstet Gynecol. 93(1), 51-58, 1999.

16. Sawaizumi T, Nanno M, Ito H: De Quervain's disease: efficacy of intra-sheath triamcinolone injection. Int Orthop. 31(2), 265-268, 2007.

17. Watanabe N, Piché M, Hotta H: Types of skin afferent fibers and spinal opioid receptors that contribute to touch-induced inhibition of heart rate changes evoked by noxious cutaneous heat stimulation. Mol Pain. 11, 4, 2015.
18. Hotta H, Schmidt RF, Uchida S, Watanabe N: Gentle mechanical skin stimulation inhibits the somatocardiac sympathetic: C-reflex elicited by excitation of unmyelinated $\mathrm{C}$-afferent fibers. Eur $\mathrm{J}$ Pain. 14(8), 806-813, 2010.

19. Watanabe N, Miyazaki S, Mukaino Y, Hotta H. Effect of gentle cutaneous stimulation on heat-induced autonomic response and subjective pain intensity in healthy humans. J Physiol Sci. 62, 343-350, 2012.

20. Mendell LM: Constructing and deconstructing the gate theory of pain. Pain. 155(2), 210-216, 2014.

21. Artioli DP, Bertolini GRF: Kinesio taping: application and results on pain: systematic review. Fisioterapia e Pesquisa. 21(1), 94-99, 2014.

22. Shinbara H, Okubo M, Kimura K, Mizunuma K, Sumiya E: Participation of calcitonin gene related peptide released via axon reflex in the local increase in muscle blood flow following manual acupuncture. Acupunct Med. 31(1), 81-87, 2013.

23. Goats GC: Massage--the scientific basis of an ancient art: Part 2. Physiological and therapeutic effects. Br J Sports Med. 28(3), 153-156, 1994.

24. Mori H, Ohsawa H, Tanaka TH, Taniwaki E, Leisman G, Nishijo $\mathrm{K}$ : Effect of massage on blood flow and muscle fatigue following isometric lumbar exercise. Med Sci Monit. 10(5), CR173CR178, 2004.

\section{Norio SAITo}

Norio SAITO graduated from Tokai University Graduate School of Science and Engineering (Japan) Master's course in 2016. He is currently a doctoral course student at the graduate school of Informatics and Engineering, The University of Electro-Communications (Japan). He works in the

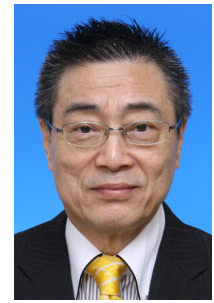
Tokai Osteopathic Institute since September 1994, after working in Hospital for 7 years. His specialty is research on pain (analgesia and tissue recovery) and he is a member of the Japanese Society for Medical and Biological Engineering.

\section{Kazuyuki Mıтo}

Kazuyuki Miтo received the $\mathrm{Ph}$. D. degree from the University of Electro-Communications, Japan, 2002. Currently, he is an associate professor in the Department of Informatics of the University of Electro-Communications. His research fields are ergonomics, welfare engineering, and Kansei engi-

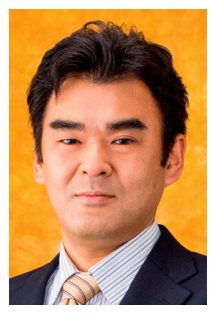
neering. He is a member of the International Society of Electrophysiology and Kinesiology (ISEK), the Institute of Electronics, Information and Communication Engineers (IEICE), and the Institute of Electrical Engineers of Japan (IEEJ). 